\title{
The effect of surface temperature on dynamics of water droplet in minichannel with gas flow
}

\author{
Ekaterina Isachenko ${ }^{1,2, *}$ and Evgeniy Orlik ${ }^{1,2}$ \\ ${ }^{1}$ Kutateladze Institute of Thermophysics SB RAS, 1 Lavrentyev Ave., 630090, Novosibirsk, Russia \\ ${ }^{2}$ Department, Novosibirsk State University, 2 Pirogova Str., 630090, Novosibirsk, Russia
}

\begin{abstract}
The experiments have been carried out to study dynamics of liquid droplets, blown by the gas flow in a mini-channel. The mean velocity at which the droplet motion over the substrate starts was determined depending on the surface temperature at different droplet volumes. The shadow method was the main method of measurement. The advancing and receding contact angles were measured depending on the gas flow rate. The friction force was determined using the advancing and receding contact angles and droplet size. A motion of a droplet was also observed from the top. The local velocity and acceleration of droplet were calculated.
\end{abstract}

\section{Introduction}

The development of microelectronics is closely related to the problem of heat removal from the semiconductor chip. To date, the most affective cooling systems are the systems based on impact jets and sprays, two-phase flows in minichannels and fluid flows in microchannels. These systems enable removal of heat fluxes of up to $500 \mathrm{~W}$ from the chip size 10 to $10 \mathrm{~mm}[1,2,3,4]$. However, already now the electronic industry is ready to produce components where the heat flux density can reach values of $1 \mathrm{~kW} / \mathrm{cm}^{2}$ and higher [6]. Even the most effective systems that use the two-phase flows are unable to cool such components, which is a technological barrier to the further development of microelectronic systems. One of the technical solutions to achieve significant intensification of heat transfer is a device, forming near-wall droplet flows in micro - and minichannels. The transition from a continuous film flow to the droplet flow with an increase in the length of the contact lines leads to the intensification of heat transfer at evaporation [7].

Above all the dynamics of a droplet in the gas flow is studied in the absence of evaporation. There are studies about dynamics of liquid droplet in turbulent flow in a sufficiently high channel comparing with height of droplet [8] and including vibration effect [9]. Numerical work [10] reports the dynamic and interaction of two droplets in a channel with gas flow, where the key parameter is the distance between the droplets. This interaction between the droplets has to be considered for designing cooling systems with forming near-wall droplet flows.

\footnotetext{
* Corresponding author: isach-e@yandex.ru
} 
Significantly, changing viscosity and surface tension in liquid droplet on heated substrate may have a significant impact on its dynamics. But there is lack of data in the literature devoted to this aspect. In the scope of present work the dynamics of single droplet has been explored depending temperature of substrate and size of droplet in mini-channel with gas flow.

\section{The experimental setup}

The scheme of the experimental setup is shown in figure 1. It includes a channel with height $6 \mathrm{~mm}$, removable substrates, the substrate temperature controlling and the air supply systems. Optical access for visualisation provided through the windows from side walls and the top cover. Ultrapure water obtained with the use of Milli-Q system is used as working fluid. The droplet is placed on surface into the channel with a syringe. The experiments have been carried out at the initial droplet volume of 60-150 $\mu$ l. All experiments were realized on a substrate of stainless steel with polished surface. The Reynolds number of the gas flow was varied from 0 to 7000 . The source gas is dry air from special air compressor. The temperature is maintained $25^{\circ} \mathrm{C}$ using a thermoregulation system. The substrate was heated by Peltier elements, installed in contact with the substrate. The substrate temperature during the experiment was regulated with a precision of $0.2^{\circ} \mathrm{C}$ using the PR-59 controller.

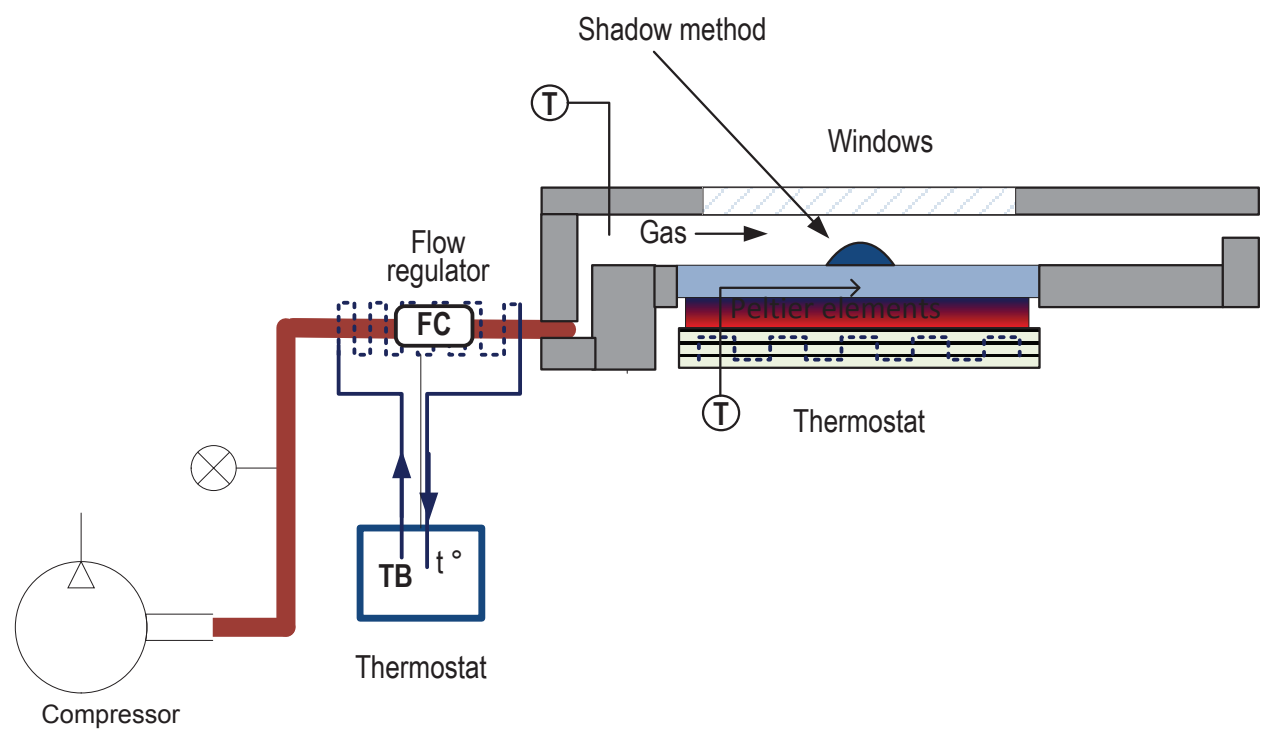

Fig. 1. Scheme of the experimental setup.

To register the contour of a sessile droplet the shadow method was used. Its principle is based on the fact that the physical object is illuminated by a parallel light beam, and its shadow is recorded by the camera, as shown in Fig. 2. Optical equipment allows obtaining images with a resolution of $6 \mu \mathrm{m} /$ pixel (see Fig. 3). The obtained images were processed by different methods with the help of software (the Drop Shape Analysis by KRÜSS). Before the experiments, the hysteresis of the contact angle on the substrate was measured. The advancing angle of wetting was around 80 degrees, and the receding angle was around 50 degrees. Images obtained above droplet were used for calculation of the local velocity and acceleration (see. Fig. 6). 


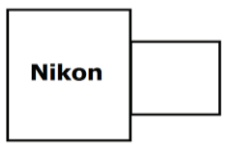

a)

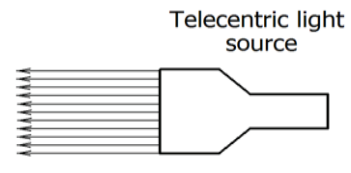

b)

Fig. 2. Shadow method - a), schematic drawing of the droplet in the channel $-b$ ).

\section{Results}

At an increased velocity of the gas flow, a droplet lost symmetry (see Fig. 3), and at some velocity values it started moving over the substrate (see Fig. 6). Flow velocity required for moving droplet decreasing with increasing of droplet volume that evidenced by the data which are presented in Figure 4. It should be noted that increasing temperature of the substrate surface, leads a decrease in the receding contact angle, in other words, the stack of droplet to the surface. One of the explanations that must be taken into consideration is that the viscosity and the surface tension of water significantly depend on temperature.

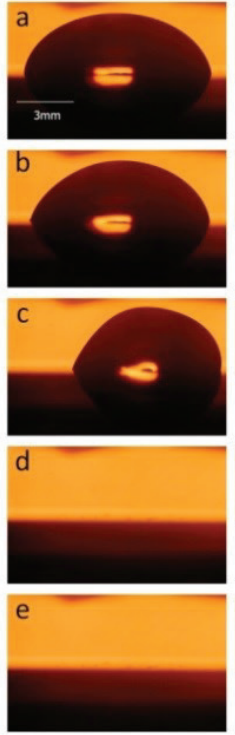

$\mathrm{T}=27 \mathrm{C}$
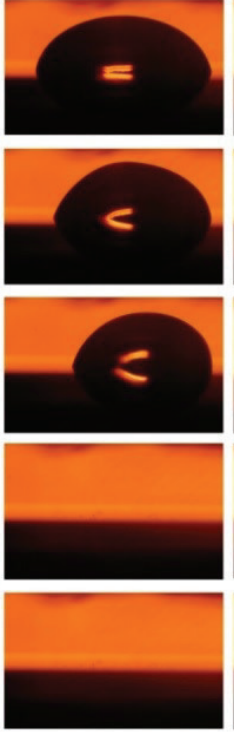

$T=50 \mathrm{C}$
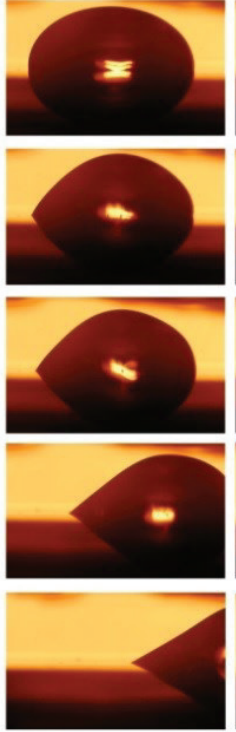

$\mathrm{T}=60 \mathrm{C}$
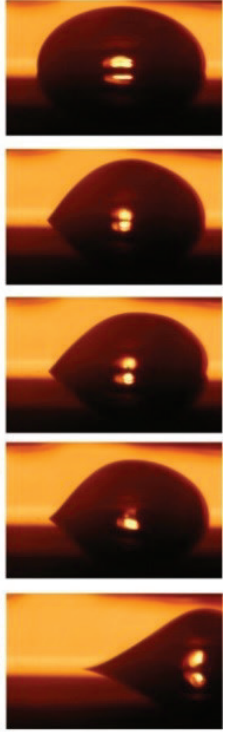

$T=70 \mathrm{C}$

Fig. 3. Droplet dynamics. Mean velocity of air in $\mathrm{m} / \mathrm{s}:$ a) 0 ,b) 4.2 , c) 4.6 , d) 5.5 , e) 6.3 . 


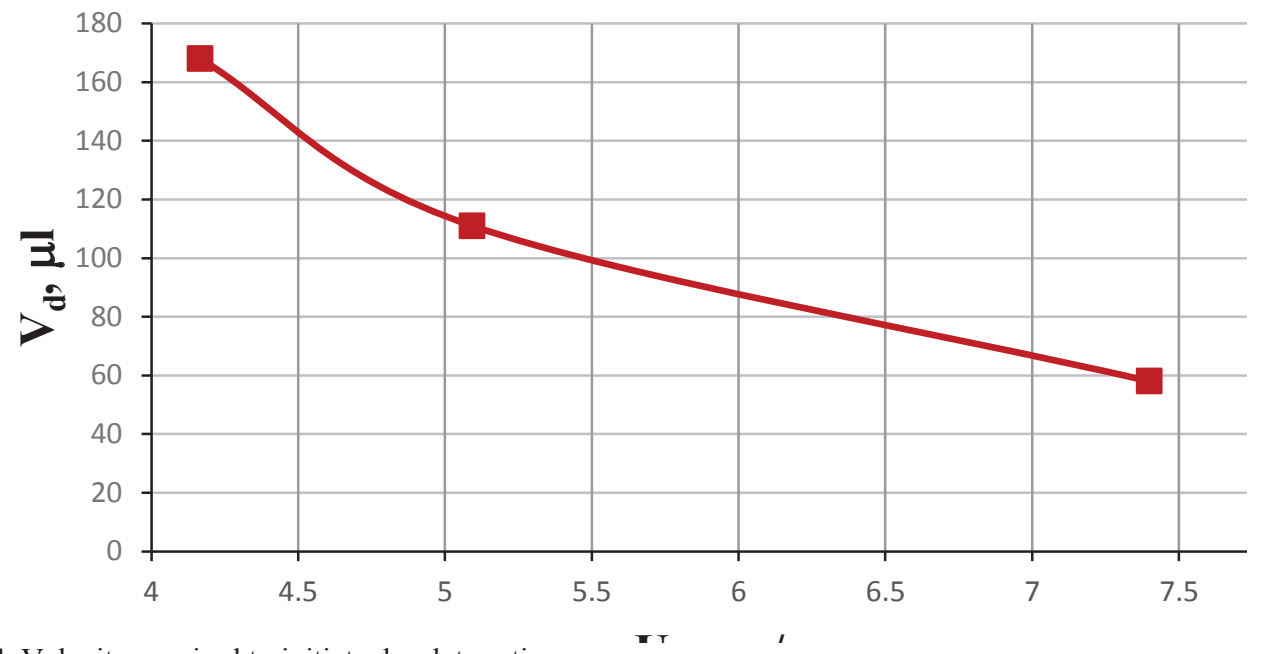

Fig. 4. Velocity required to initiate droplet motion.

Figure 5 shows the friction force affecting the contact line. It was determined by the formula $2 \sigma r(\cos (A)-\cos (R))$, where $\sigma$ is the surface tension, and the angles $A$ and $R, r$ is radius as are shown in Fig. 2 b). The larger symbols correspond to the values at which the droplet began its motion. In the work [8] the dynamics is studied for different droplets on the silane substrates. The values of velocity required to move droplet and correspondent friction forces in [8] were very similar to those obtained in our work. In our work it was found that with increasing temperature the droplet motion starts at somewhat larger gas flows, and the friction force affecting the droplet on the heated surface, calculated by the above formula, is a few times larger than in the case without heating.

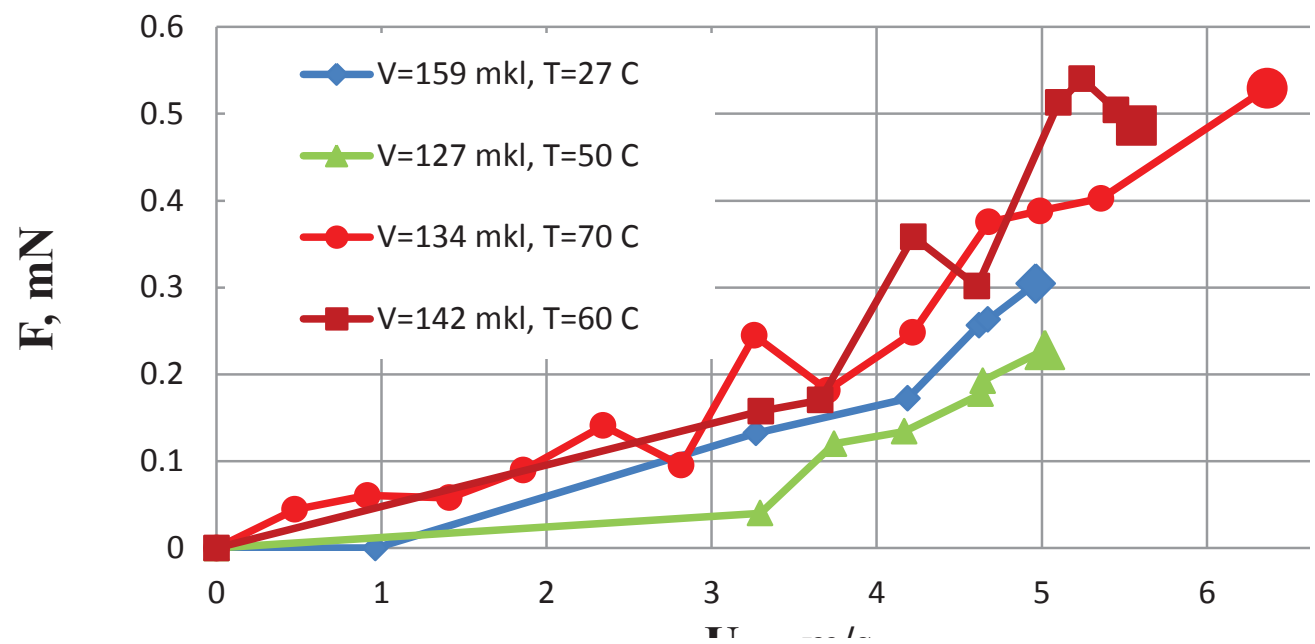

Fig. 5. Friction force affecting the droplet contact line, depending on the air flow rate and substrate temperature.

A local velocity rate of water droplet was determined using analysis of images, which were recorded from top. After droplet was starting movement, its velocity reaches about $1 \sim 2 \mathrm{~mm}$ per second for $50 \mathrm{~mm}$ path, as shown in Figure 7. Droplet can locally stick during 
motion, that reduces its velocity (that is visible from figure, 40 seconds, $\mathrm{T}=27$, Figure 6 ), but the shape of droplet stays sufficiently round

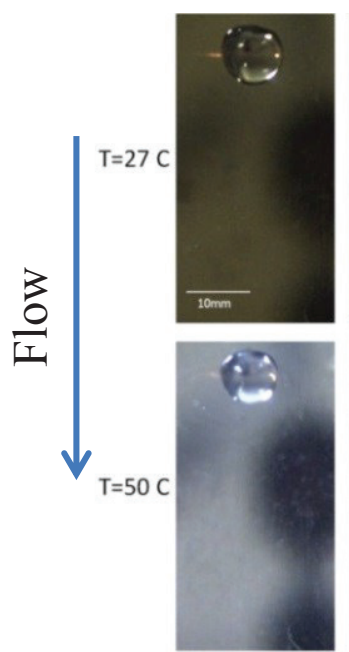

$0 \mathrm{sec}$
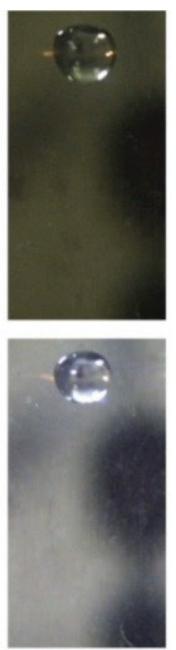

$10 \mathrm{sec}$
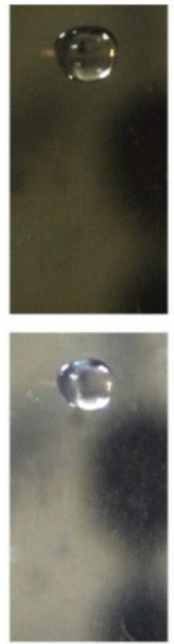

$20 \mathrm{sec}$
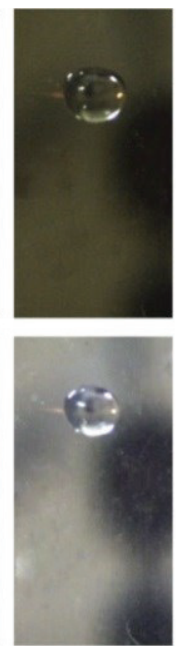

$30 \mathrm{sec}$
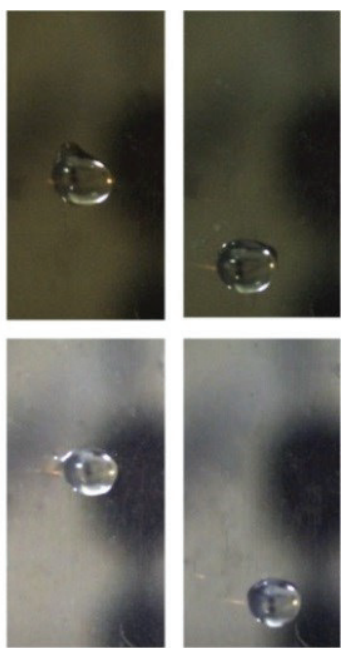

$40 \mathrm{sec}$

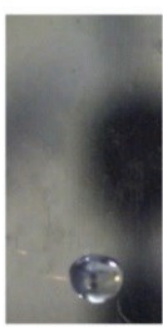

$50 \mathrm{sec}$

Fig. 6. Series of images of the movement of droplet on surface at temperature $\mathrm{T}=27 \mathrm{C}$ and $\mathrm{T}=50{ }^{\circ} \mathrm{C}$ (top view). The arrow indicates the direction of the gas flow.

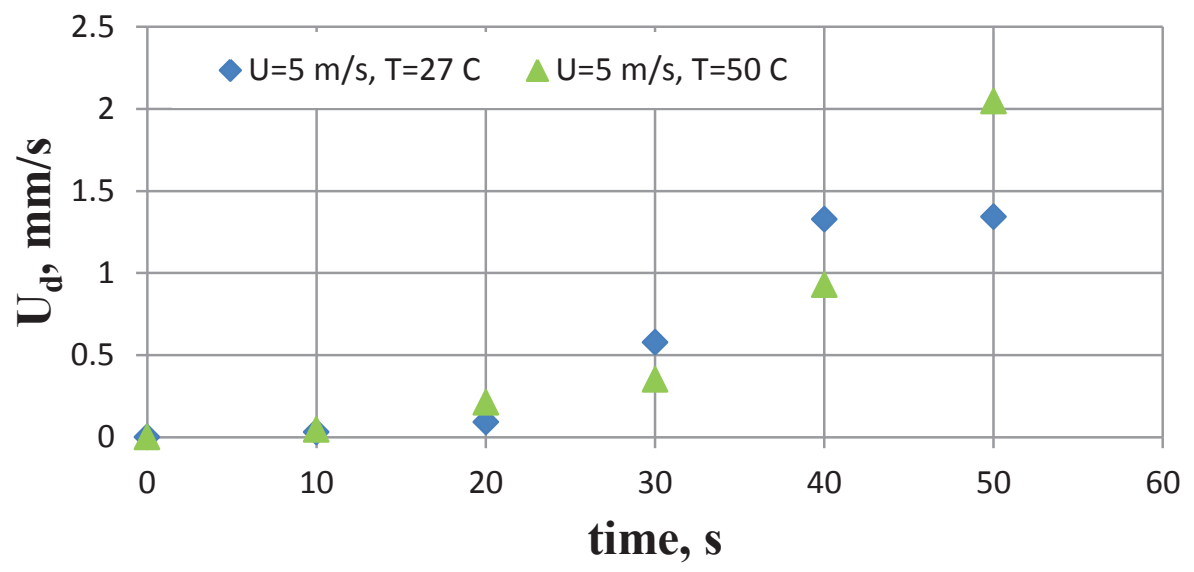

Fig. 7. $=$ Velocity of droplet calculated from figure 6.

\section{Conclusion}

In this paper, we have carried out a detailed study of the dynamics of a single droplet in the $6 \mathrm{~mm}$ channel with gas flow. New data on the dynamics of water drops on a heated surface have been obtained. It has been found that with increasing temperature, the droplet starts to stick to the substrate surface.

The authors express their gratitude to the Russian Science Foundation for the support of this work (project No. 14-19-01755). 


\section{References}

1. A. Chinnov and O. Kabov, High Temperature, 42, 2 (2004)

2. D. Zaitsev, O. Kabov, V. Cheverda and N. Bufetov, High temperature, 42, 3 (2004)

3. T. Hirokawa, H. Ohta and O. Kabov, Interfacial Phenomena and Heat Transfer, 3, 3 (2015)

4. D. Zaitsev, D. Rodionov and O. Kabov, Technical Physics Letters, 35, 7 (2009)

5. K. Jungho, International Journal of Heat and Fluid Flow, 28, 4 (2006)

6. A. Bar-Cohen, Intrachip/interchip enhanced cooling fundamentals DARPA, Microsystems Technlogy Office (2012)

7. V. Cheverda, I. Marchuc, A.Karachevsky, A. Orlik and O. Kabov, Thermophysics and Aeromechanics, 23, 3 (2016)

8. J. Fan, M. Wilson and N. Kapur, Journal of Colloid and Interface Science, 356 (2011)

9. T. Maurer and U. Janoske, Computational Methods in Multiphase Flow, 8 (2015)

10. Jie Wu and Jun-Jie Huang, Journal of Applied Physics, 118, 044902 (2015) 